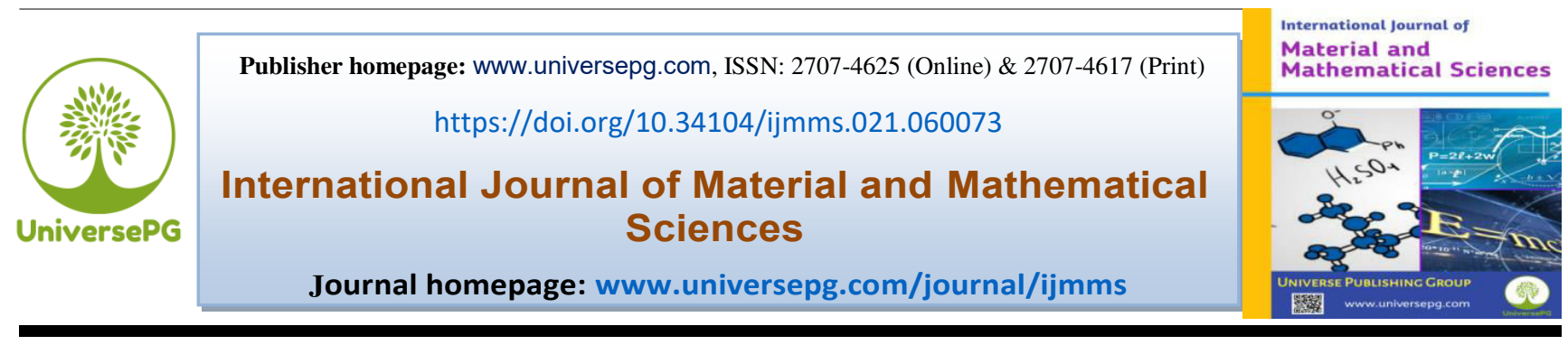

\title{
Numerical Investigation of Dropwise Condensation on Smooth Plates with Different Wettability
}

\section{Danuska Pathiranage*}

School of Computing, Engineering and Mathematics, Division of Engineering and Product Design, Brighton University, United Kingdom.

*Correspondence: danuska.pathiranage@gmail.com (Danuska Pathiranage, School of Computing, Engineering and Mathematics, Division of Engineering and Product Design, Brighton University, United Kingdom).

\begin{abstract}
Condensers are used in various energy-intensive processing industries. Improving their efficiency plays a crucial role in the optimization of energy consumption. Dropwise Condensation is a highly attractive form of heat transfer. This project investigates the effects of different wettability characteristics in dropwise condensation on smooth plates. It involves a Volume of Fluid (VOF) based Computational Fluids Dynamics (CFD) model to carry out the simulations in Open FOAM on smooth plates various wettability's. Different parameters such as receding angle, advancing angle, and various radii of droplets have been used for investigation. A base case with droplets' radius of $12.5 \mu \mathrm{m}$, receding angle, and advancing angles of $\left(34^{\circ}, 90^{\circ}\right)$ was used. Three sets of simulations were performed. In Set 1 , the angles were $\left(34^{\circ}, 90^{\circ}\right)$. The radius of the first droplet was kept constant at $12.5 \mu \mathrm{m}$ and the radius of the second droplet changes 1:4 progressively. In Set 2 , the angles are changed to $\left(154^{\circ}, 162^{\circ}\right)$, and the radius of the second droplet changes in the same manner above. In Set 3 , the angles were kept at $\left(107^{\circ}, 117^{\circ}\right)$. The radius changes as above. In some cases, the droplets merge and condense. In others, the momentum created by merging of the droplets creates repeated oscillation or even a lift-off, from the cooling plate.
\end{abstract}

Keywords: Numerical investigation, Open FOAM, Smooth plates, Dropwise, and Weight wettability.

\section{INTRODUCTION:}

Condensers are used in various energy intensive processing industries such as refrigeration, power generation and material manufacturing. Their efficiency improvement has a crucial role not only in optimazation of energy consumption, but also in protecting the environment. Enhancement of this equipment requires full knowledge of the condensation process, which is in conjunction with various phenomena such as heat transfer, phase change, and fluid dynamics. Occurrence of different types of condensation process; filmwise and dropwise, depends on existing operational and condensing surface conditions. The work by Mei et al. (2009) in the early 1930's showed that the dropwise condensation is more attractive form of heat transfer (Incropera et al., 2002) due to its much higher surface heat transfer coefficient than the film wise condensation. Advanced condenser designs can reduce device size and pressure drop significantly increasing the energy efficiency. However, such flow patterns cannot be predicted accurately using analytical or simplified models and experimental device design and optimization is often prohibitively costly. Therefore, considerable amount of research efforts has been applied in recent decades to develop computational software tools (e.g., CFD models) in order to simulate and resolve two-phase condensing flows under different operational conditions and geometries. In this project, a VOF based CFD model is used to carry out simulations on a user modified solver of 
Open FOAM (Openfoam, 2019). For drop wise condensation on smooth plates with various wet ability's.

A series of parametric simulations were carried out varying the wettability of the smooth plate. The effect of different parameters such as contact angle; advancing and receding angles; droplet radius and saturation temperature will be investigated. Dropwise condensation process begins with low nucleation sites, also known as the physical separation of gas, liquid and solid, early stage of phase change due to condensation. Droplets grow by condensation and coalescence with the other droplets till the droplets have grown enough to be removed from the surface by merging and sliding. Once the droplets are removed, the exposed portion of the surface allows to form new droplets and be nucleated (Rose, 1981), thus the dropwise condensation process to continue in a cyclic steady state. Coalescence is the fundamental mode of condensing droplets that are bigger than (Zemach et al., 1992), the smallest thermodynamically viable droplet (e.g., Radius $r>2 u m$ for water at $1 \mathrm{~atm}$ ) but no previous studies has investigated the direct effect of different wettability characteristics on the phenomena. During the process of the coalscence, the droplets may rumble on the surface and continue to condense. Additionally, in some other cases, due to the momentum created by the merging of the droplets, they may oscillate repeatedly and clear off the surface area. The two events occur depending on the characteristics of the surface. Most influential process that occurs in a dropwise coalscence is condensation. Condensation will allow small amounts of water resulted through reduction of temperature between the main surface and its ambient surface temperature (Chu et al., 2012). Change from its surface heat transfer efficiency has become a key aspect resulted through the dropwise condensation occurred through surfaces, natural or artificial. Coalescence results in two different forms of masses combining together, in this case droplets of water. Which is resulted between two pressures from the droplets from its initial stage and pressure from its final destination, in this case a wet surface. Dropwise calescence is proved to be a common factor for heat exchange between two surfaces that has a temperature change in between the two. There are several characteristics and energy transfers that occur throughout the dropwise coalescence- kinetic energy to gravitational potential energy results from the movement of the droplet to the differed surface. Larger drops that coalescence results from its neighbouring molecules that combine together and tend to become larger in size. Gradually the droplet will reach its maximum weight that it could handle itself and become unstable allowing the larger molecule to break off the initial surface ground on its secondary surface resulting in a heat exchange from kinetic to thermal exchange (Jalil and Karami, 2020; Chu et al., 2012).

The coalescence of droplets in dropwise condensation has been a topic studied and researched by many in the recent past. While contact angle hysteresis and wettability of surface on the coalescence of droplets have been studied by Chu et al. (2012) and Somwanshi et al. (2014), a framework has also been further developed on the basis of this study as well as the disappearance of the interface internally that takes place gradually (Sprittles and Shikhmurzaev, 2012; Enright et al., 2014; Chen et al., 2016) have used the numerical framework for the droplets to jump on adhesion surfaces that are low, induced by model coalescence. Further, there have also been experiments conducted on surfaces that are resistant to water with certain roughness to study the same (Mei et al., 2013). Such scholarship has helped improve the understanding of droplet coalescence of hydrodynamics; however, the studies on the transfer of heat takes place during such coalescence have not been as much. Simulations have been carried out numerically however, wherein the single droplets are studied in a Lagrangian sense (Burnside et al., 1999). The investigations carried out so far have been responsible in expressing droplet coalescence as an immediate event. It is safe to say that these models consist of two droplets merging to become one large droplet and the volume is ideally conserved with the centre at the mean of the weight of the volume of the two previous centres. The heat transfer that takes place at the next stage of the process contains the newly formed droplet to recover from temperature due to collusion as well as the heat transfer rate. This however, does not take into account the unsteadiness of the hydrodynamics in coalescence. The time would vary in reality for each droplet during coalescence, depending on the shape of its base. Droplets that oscillate are also known to affect the area around them. There are thus many such factors that are unaccounted for, that impact the overall rate of heat transfer. Response time and spatial and temporal scales at such initial stages of coalescence process may prove it carry out experiment. For example, the present work uses drop 
radius varying from $(12.5 \mathrm{~mm} \leq \mathrm{r} \leq 100 \mathrm{~mm})$ and time scale of (oms $\leq \mathrm{t} \leq 500 \mathrm{~ms}$ ). Observing these stages can prove to be extremely challenging; hence there are not many studies to validate the effect of heat transfer during condensation.

1.1 Project Management - Appendix 7.2 shows the initial Gantt chart that was designed to follow up tasks throughout the year of final year study. There were many moments that made the project very interesting and enthusiastic to work towards the objectives. However, many setbacks were found upon throughout the year. With the guidance of the supervisor, the setbacks were tackled, and the project was set back on track. During the first few weeks the tasks were complete and everything was on track. However, due to the rush into the holidays and the time constraint that was available during the Easter break, the second simulation was not able to be done on time (shown in red on Gantt chart, Appendix 7.2). Most tasks were finished on time but the delay on the second simulation created a major problem towards the end of the academic year even though the project was due to finish at the start of April, the second simulation resulted in a 3-week set-back. However, the issue was resolved and the simulation was completed successfully on time with set of results and the project objectives were accomplished; i) Numerical Method, and ii) Governing equations. The numerical method including the governing equations and phase change model are in this section. Both liquid and vapour phases are treated as incompressible, Newtonian fluids. The mass conservation equation is as follows: Equation 1 (Georgoulas et al., 2017).

$$
\nabla \cdot(\rho \vec{u})=\dot{\rho},
$$

The phase change is accounted for by the source term $\dot{\rho}$ on the right-hand side. The mass is also globally conserved since the mass which is removed from the liquid side of the interface is added on the vapour side. Conservation of momentum is given as follows: Equation 2.

$$
\frac{\partial}{\partial t}(\rho \vec{u})+\nabla \cdot(\rho \vec{u} u)-\nabla \cdot\left\{\mu\left[\nabla \vec{u}+(\nabla \vec{u})^{T}\right]\right\}=-\nabla p+\vec{f}_{S T}+\vec{f}_{g^{\prime}}
$$

The effect of surface tension and gravity are respectively accounted for on the momentum source terms at the right side of the equation. The surface tension is modelled according to the continuum method (Deshpande et al., 2012). Conservation of energy is given as follows: Equation 3 (Antonini et al., 2013).

$$
\frac{\partial}{\partial t}\left(\rho c_{p} T\right)+\nabla \cdot\left(\vec{u} \rho c_{p} T\right)-\nabla \cdot(\lambda \nabla T)=\dot{h},
$$

Where bulk heat capacity is represented by term $c_{p}$, (T) temperature, and bulk thermal conductivity by term $\lambda$. The volume fraction $\alpha$ is transferred by the flow field in the equation given below. The sharpening of the interface is vital when simulating two phase flows of fluids. This is artificially obtained in OpenFoam through the introduction of extra compression term (V. $\left(\alpha(1-\alpha) U_{r}\right)$ in the equation (4). Equation 4 (Somwanshi et al., 2014),

$$
\frac{\partial \alpha}{\partial t}+\nabla \cdot(\alpha \vec{U})-\nabla \cdot\left(\alpha(1-\alpha) U_{r}\right)=\frac{\dot{\rho}}{\rho} \alpha,
$$

The artificial compression velocity $U_{r}$ in equation 4 is calculated using the following equation: Equation 5 (Georgoulas et al., (2017)

$$
u_{r}=n_{f} \min \left[C_{\gamma} \frac{|\varphi|}{\left|S_{f}\right|}, \max \left(\frac{|\varphi|}{\left|S_{f}\right|}\right)\right] .
$$

Where the cell surface normal vector represented by term $n_{f}$, mass flux by f, surface area of a cell by $S_{f}$ and $C_{\gamma}$ is a coefficient where the value is set between 1 to 4 . The relative velocity of the two fluid phases across the interface is represented by term $U_{r}$. In equation (4), the conservation of the volume fraction $\alpha$ is ensured by the process of compression velocity $U_{r}$, the term $\alpha(1-\alpha)$ limits this artificial compression approach only in the vicinity of the interface, where $0<\alpha<1$. The level of compression is dependent on the value of $C_{\gamma}$ (Hoang et al., 2013 and Georgoulas et al., 2017). VOF model used for this investigation in OpenFOAM does not solve equation (4) completely, instead applies a multi-dimensional universal limiter with an explicit solution algorithm in order to achieve a sharp interface and limits the volume fraction between 0 and 1 (Georgoulas et al., 2017). The average over the liquid and vapour phases, weighted with the volume fraction are computed as bulk properties. The following equation explains the process: Equation 6 (Georgoulas et al., 2017),

$$
\gamma=\alpha \gamma_{l}+(1-\alpha) \gamma_{\nabla}
$$

The VOF method is known to be affected by currents that are non-physical and spurious in the region of the interface. This may be owing to errors in normal vector calculation and interface curvature used. Such errors can be accounted for using the following method: Equation 7 (Georgoulas et al., 2017),

$$
\kappa=\nabla \cdot\left(\frac{\nabla \widetilde{a}}{|\nabla \widetilde{a}|}\right) \text {. }
$$




\subsection{Phase Change model}

The following equation has been used to calculate the evaporating mass flux at the liquid-vapour interface: Equation 8 (Georgoulas et al., 2017). The following equation was used to calculate the interfacial heat resistance: Equation 9 (Georgoulas et al., 2017)

$$
j_{\text {evap }}=\frac{T_{\text {int }}-T_{\text {sat }}}{R_{\text {int }} h_{\text {lv }}}, \quad R_{\text {int }}=\frac{2-\gamma}{\gamma} \frac{\sqrt{2 \pi R_{\text {gas }}}}{h_{\text {lv }}^{2}} \frac{T_{\text {sat }}^{3 / 2}}{\rho_{v}},
$$

The uncertainty of the parameter $\gamma$ which lies in the range of 0 and 1 and is considered as the evaporation or condensation coefficient. The amount of liquid evaporated is locally calculated and numerical instabilities are avoided by smearing the resulting source term field over a few cells. Equation 9 calculates the evaporating/condensing mass flux and is incorporated in the conservation equations. This can be seen in the equation: Equation 10 (Georgoulas et al., 2017)

$$
\dot{\rho}_{0}=j_{\text {eodap }}|\nabla a| \text {, }
$$

The following equation is used for the Net Mass Flow: Equation 11(Georgoulas et al., 2017)

$$
\dot{m}_{\text {int }}=\iiint \dot{\rho}_{0} \mathrm{~d} V,
$$

The diffusion equation for the smooth distribution of source terms: Equation 12 (Georgoulas et al., 2017).

$$
\dot{\rho}_{1}-\nabla \cdot\left[(D \Delta \tau) \nabla \dot{\rho}_{1}\right]=\dot{\rho}_{0},
$$

Neuman boundary conditions are imposed for the smooth source term field on all boundaries of the domain. This is to ensure that both integral values of the sharp and smooth source fields remain the same, even after the smearing. Further, the width of the source term field is proportional to the square root of the product of the diffusion constant (adjusted to the mesh resolution for the smearing of the source field on several cells) and the artificial time step. Those source terms in all cells without pure liquid or vapour are set to zero, artificially. By doing so, source terms are shifted into the pure vapour and at the vicinity of the interface remain liquid cells. Thus, velocity field which is calculated is used to transport the interface, in the absence of any source terms. The algorithm for transport for volume fraction field and compression of the interface that is associated works in an efficient manner without the source term field interfering. The source term field which remains is scaled individually on the liquid and vapour side through appropriate scaling coefficients. Mass will thus be globally conserved through this and the evaporating/condensing mass flow corresponds to the net mass glow globally through the interface. These scaling coefficients can be obtained through integration of the smooth source term field in each of the phases that are pure and by comparison to the net mass flow. The following equations can explain these factors: Equation 13 and 14 (Georgoulas et al., 2017)

$$
\begin{gathered}
N_{1}=\dot{m}_{\text {int }}\left[\iiint\left(\alpha-1+a_{c u t}\right) \dot{\rho}_{1} \mathrm{~d} V\right]^{-1}, \\
N_{v}=\dot{m}_{\text {int }}\left[\iiint\left(a_{\text {cut }}-\alpha\right) \dot{\rho}_{1} \mathrm{~d} V\right]^{-1},
\end{gathered}
$$

The above are useful scaling factors in obtaining the final source term distribution through the following process: Equation 15 (Georgoulas et al., 2017).

$$
\dot{\rho}=N_{v}\left(\alpha_{\text {cut }}-a\right) \dot{\rho}_{1}-N_{l}\left(a-1+\alpha_{\text {cut }}\right) \dot{\rho}_{1},
$$

This can be illustrated through the diagram below:

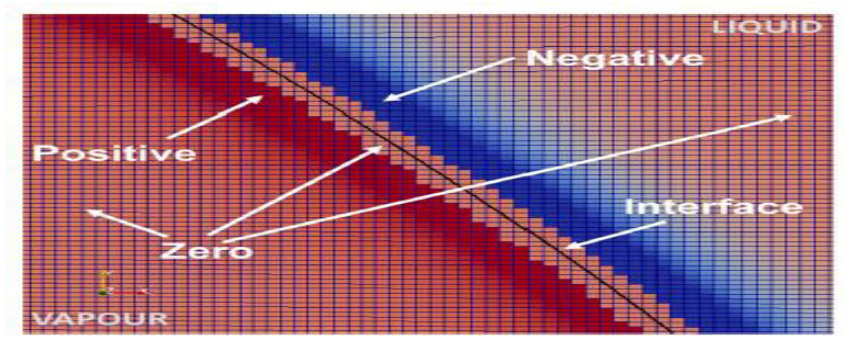

Fig 1: The diagram depicts an evaporating bubble, where in the distribution of the final source terms can be viewed in the computational domain (Georgoulas et al., 2017).

The final equation brings about the source term: Equation (16).

$\dot{h}=\left[N_{v}(1-\alpha) C_{p v}-N_{l} \alpha C_{p l}\right] \dot{\rho}_{1} T-\dot{\rho} h_{l v}$,

The heat capacity is represented by equation (16). While the correction source term responsible for removing artefacts, is explained through the first part; the contribution of the enthalpy is explained through the second part. VOF Solution algorithm used in OpenFOAM summarised in a flowchart in Fig 2.

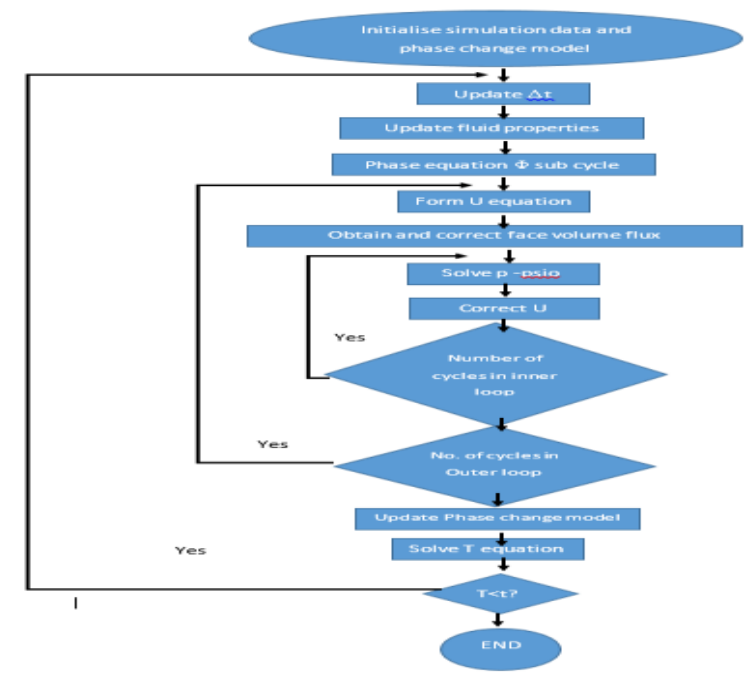

Fig 2: VOF Solution Algorithm. 


\section{Simulation Studies}

2.1 Computational Domain \& Mesh - As the coalescence and condensation process of the droplets in the investigation is considered symmetric, a symmetric computational domain was constructed for the investigation. Fig 2 illustrates the representative domain with labels. The constructed computational domain is rectangular cuboids with length, width and height varying in each case. Here, $\mathrm{H}=\mathrm{W}=\left(2 \mathrm{x} \mathrm{r}_{1}+\right.$ Rt $\left.x r_{1}\right)$ and $L=\left(6 \times r_{1}+2 \times R t \times r_{1}\right), r_{1}$ is radius of the smaller droplet, and Rt $\geq 2$ and changes progressively; is the ratio of the droplets, these values can be found on Table 2 in section 3.3 numerical simulation details below. Computational mesh consists of 144400 hexahedral cells, for the base case.

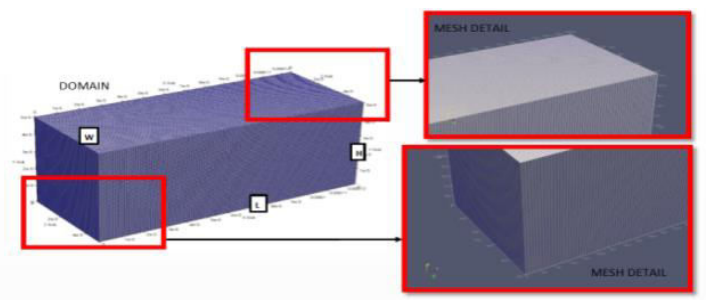

Fig 3: Illustrates the symmetric computational domain and mesh.

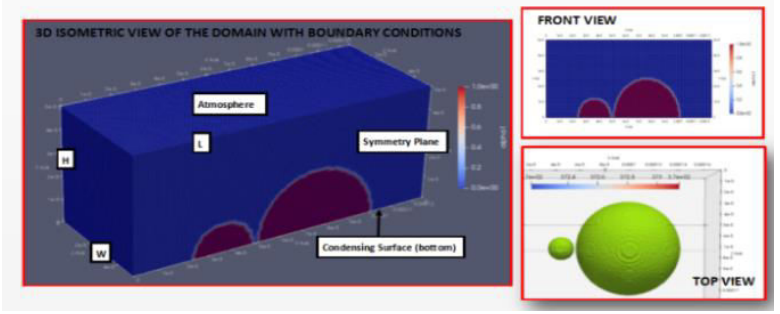

Fig 4: illustrates representative domain for base case in the investigation.

Fig 3 illustrates representative domain for case 1 (Georgoulas et al., 2017). The domain only includes half of the droplets and their surroundings, as two droplets are symmetric along one centre plane. The face illustrating the two droplets is the symmetry plane; the bottom surface is the condensing surface; and the remaining faces are open atmosphere. As illustrated in Fig 3, atmospheric values for volume fraction, $\mathrm{t}, \mathrm{U}$ and $\mathrm{P}$ are kept at zero gradient.

\subsection{Boundary conditions}

Table 1: Shows boundary conditions for the simulation domain for the present investigation (Initial Boundary conditions).

$\begin{array}{ll}\text { Boundary } & \text { Boundary Condition } \\ \text { Symmetry } & \text { Symmetry plane } \\ \text { Atmosphere } & \text { alpha, } T, U, P=\text { Zero gradient } \\ \text { Condensing Surface } & T_{\text {Sub }}=\left(T_{\text {sat }}-1 \mathrm{~K}\right)=(373.15- \\ & \begin{array}{l}\mathrm{U}=372.15 \mathrm{~K} \\ \mathrm{U}, \text { aplha, } \mathrm{P}=\text { Zero gradient }\end{array}\end{array}$

UniversePG I www.universepg.com

\subsection{Numerical simulation details}

In order to investigate the effect of surface wettability, number of simulations (series A) with different parameters such as receding angle, advancing angle and various radii of droplets have been used as shown in Table 2.

\begin{tabular}{|c|c|c|c|c|c|c|c|c|c|c|c|c|}
\hline \multicolumn{2}{|c|}{$\begin{array}{l}\text { Table } 2 \\
\text { Series A }\end{array}$} & $\mathrm{rl}(\mu \mathrm{m})$ & $\mathrm{r} 2(\mathrm{um})$ & Rt & $\mathrm{L}(\mathrm{x})$ & $\mathrm{H}(\mathrm{y})$ & $\mathbb{W}(z)$ & $\operatorname{Sid}(u \mathrm{~m})$ & $\mathrm{A} / \mathrm{a}$ & $\mathrm{R} / \mathrm{a}$ & $\begin{array}{c}\text { Centre of } \\
\mathrm{rl}(u \mathrm{~m})\end{array}$ & $\begin{array}{c}\text { Centre of } \\
\mathrm{r} 2(\mu \mathrm{m})\end{array}$ \\
\hline \multirow{3}{*}{ SET I } & CASE 1 & 12.5 & 25 & 2 & 125 & 50 & 50 & 0.2 & \multirow{3}{*}{$90^{\circ}$} & \multirow{3}{*}{$34^{\circ}$} & \multirow{3}{*}{36.5} & 76 \\
\hline & CASE 2 & 12.5 & 50 & 4 & 175 & 75 & 75 & 0.2 & & & & 101 \\
\hline & CASE 3 & 12.5 & 100 & 8 & 275 & 125 & 125 & 0.2 & & & & 151 \\
\hline \multirow{3}{*}{ SET ? } & CASE 4 & 12.5 & 25 & 2 & 125 & 50 & 50 & 0.2 & \multirow{3}{*}{$162^{\circ}$} & \multirow{3}{*}{$154^{\circ}$} & \multirow{3}{*}{36.5} & 76 \\
\hline & CASES & 12.5 & 50 & 4 & 175 & 75 & 75 & 0.2 & & & & 101 \\
\hline & CASE 6 & 12.5 & 100 & 8 & 275 & 125 & 125 & 0.2 & & & & 151 \\
\hline \multirow{3}{*}{ SET 3} & CASE 7 & 12.5 & 25 & 2 & 125 & 50 & 50 & 0.2 & \multirow{3}{*}{$117^{\circ}$} & \multirow{3}{*}{$107^{\circ}$} & \multirow{3}{*}{36.5} & 76 \\
\hline & CASE \& & 12.5 & 50 & 4 & 175 & 75 & 75 & 0.2 & & & & 101 \\
\hline & CASE9 & 12.5 & 100 & 8 & 275 & 125 & 125 & 0.2 & & & & 151 \\
\hline
\end{tabular}

S/d-bubble separation, A/a-Advancing angle, R/a-Receding angle, Rt-Ratio

In the present work simulations, a radius of $12.5 \mu \mathrm{m}$ for the first droplet kept constant throughout the investigation. Three sets of simulations were performed altering the wettability by changing advancing and receding angles. In Set 1 , the angles were $\left(34^{\circ}\right.$, $90^{\circ}$ ). The ratio (Rt) is given on Table 2 , $\mathrm{Rt}$ is the ratio of which the second droplet's radius increases in each case. The angles were (Antonini et al., 2013) changed to $\left(154^{\circ}, 162^{\circ}\right)$ which has been used and validated in previous work done regarding the droplet receding angles and the radius changes as above. In Set 3, the angles were (Antonini et al., 2013) kept at $\left(107^{\circ}, 117^{\circ}\right)$. The radius changes as above. The time of the simulations were limited to $500 \mathrm{~ms}$ in order to observe the initial phase of dropwise condensation

\section{RESULTS AND DISCUSSION:}

\subsection{Effect of different surface wettability and dif- ferent droplet ratio-Series A}

In the current section of the present paper, the effect of surface wettability characteristics on droplet condensation is investigated numerically. Simulation series A, three sets of simulations with nine cases in total were carried out varying parameters explained in Table 2. The results of the simulations were as follows. In summary, set 1, Case 1-3 simulations were carried out with the same contact angles, which mean the surface wettability was kept constant. A receding angle of $34^{\circ}$ and an advancing angle of $90^{\circ}$ creating a hydrophilic condition on the surface. However, the second droplet size was altered with regard to values Rt in Table 2. 

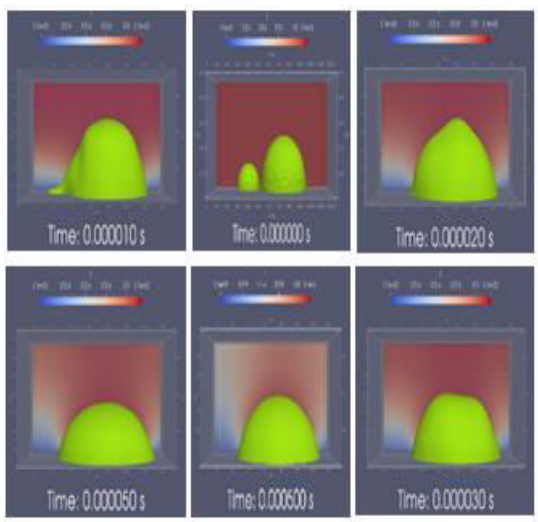

Fig 5: Effect of different contact a different radius of droplets for Case 1.

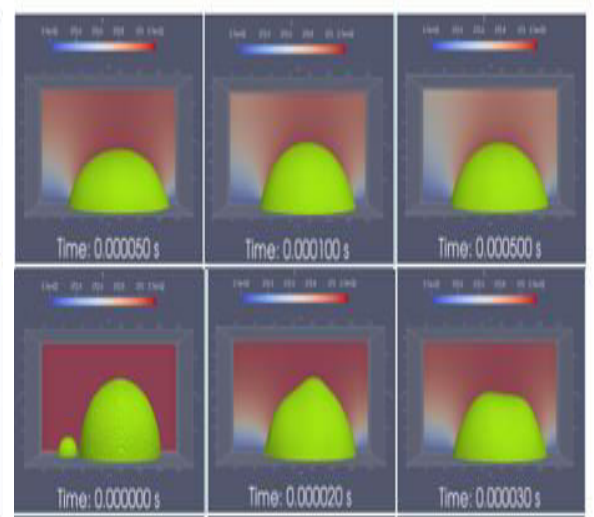

Fig 6: Effect of different contact angle and different radius of droplets Case 2.
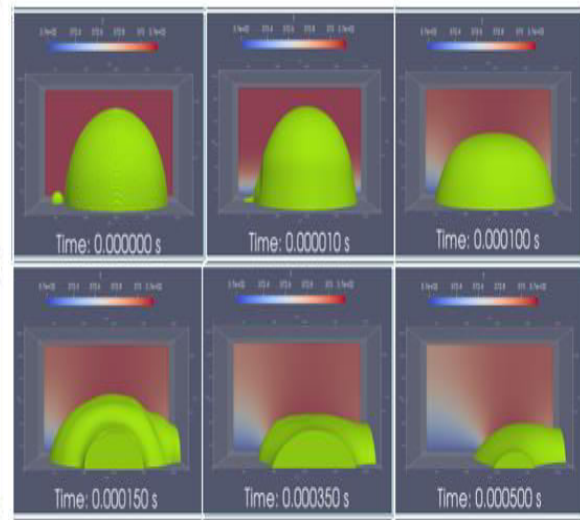

Fig 7: Effect of different contact angle and different radius of droplets for Case 3.

Results show these droplets undergo the primary mode of coalescence, where the two droplets merge together with time forming a single droplet with a larger mass and condense gradually. This also shows the effect of various droplet radii. In case 1 and 2 the droplets merger and condense slowly, where as in case 3 the droplets merge and condense at a much faster rate given the same time proving the droplet size effect the condensation rate. Set 2, Case 4-6 were carried out with a different contact angle to that of Set 1 altering the wettability of the condensing surface. A receding angle of $154^{\circ}$ and an advancing angle of $162^{\circ}$ creating a super hydrophobic condition on the surface. The aspect ratio of the droplets alters as

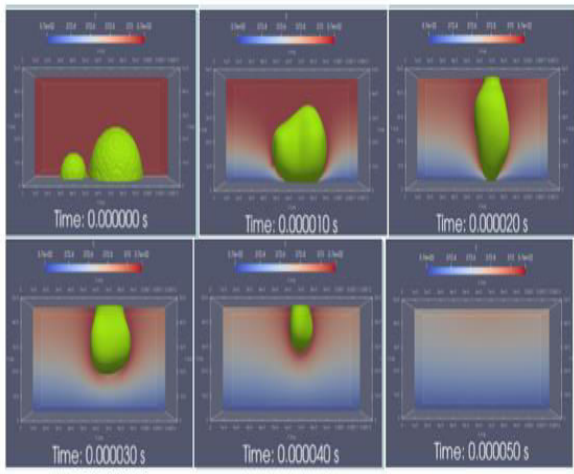

Fig 8: Effect of different contact angle and different radius of droplets for Case 4.
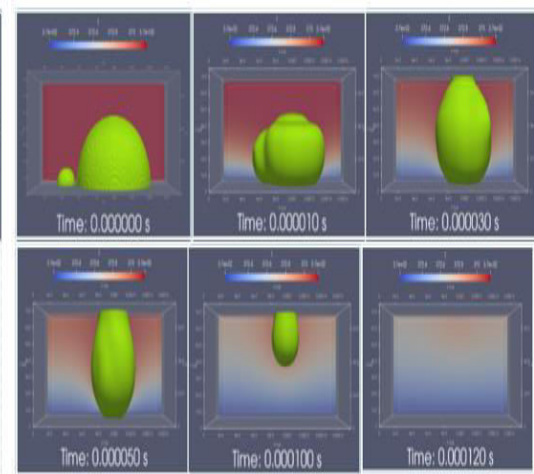

Fig 9: Effect of different contact angle and different radius of droplets for Case 5 .

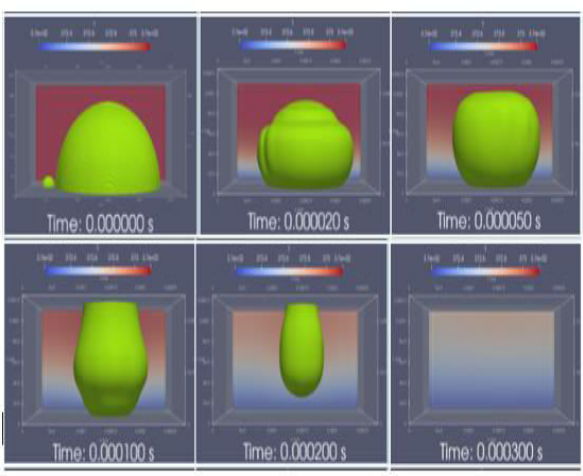

Fig 10: Effect of different contact angle and different radius of droplets for Case 6.
In this Set 3, Case 7-9, were performed with different contact angles altering the wettability of the condensing surface. A receding angle of $107^{\circ}$ and an advancing angle of $117^{\circ}$ creating a hydrophobic condition on the surface. The aspect ratio of the droplets alters as in Set 1 and Set 2. Results in these cases shows that they undergo a similar process to case 1-3. However, the momentum created by merging of the two droplets were insufficient to overcome the UniversePG I www.universepg.com surface tension and lift off, hence the merged droplets gradually condense with time. Once again, it can be observed that the size of the droplet plays a vital role in the initial phase of condensation as the droplet with larger radius condenses quicker than the two smaller droplets. Simulation series (A) was successfully completed and applicable data was obtained. Results obtained in the present work showed varying wettability have a significant effect on the dynamics of 
the two droplets after the coalescence points. This will be further discussed with a more quantitative should significantly affect the transient condensation analysis by comparing the transient condensation rate rate during the coalescence process; however, this during the phenomenon (Hasan et al., 2020).

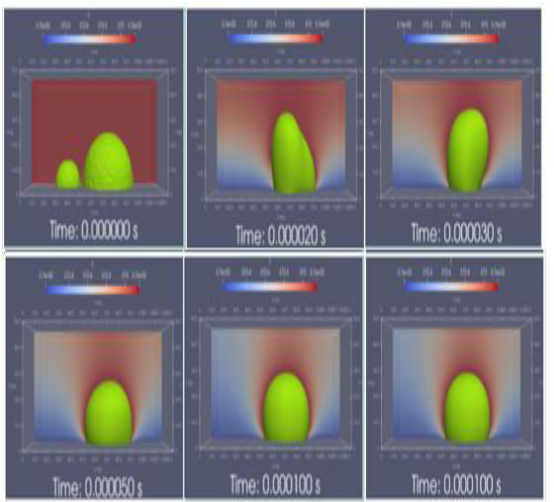

Fig 11: Effect of different contact angle and different radius of droplets for Case 7.

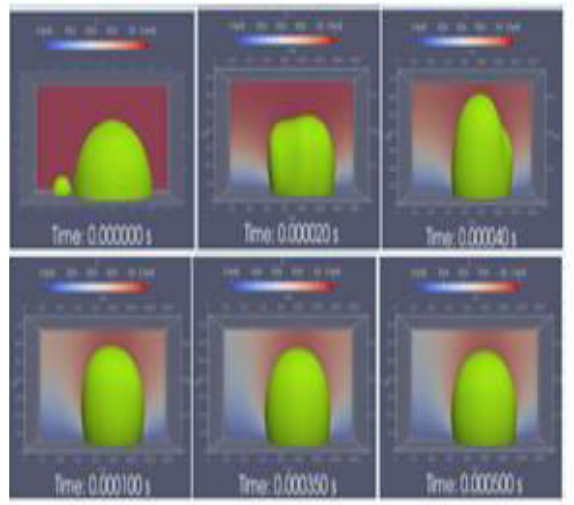

Fig 12: Effect of different contact angle and different radius of droplets for Case 8.

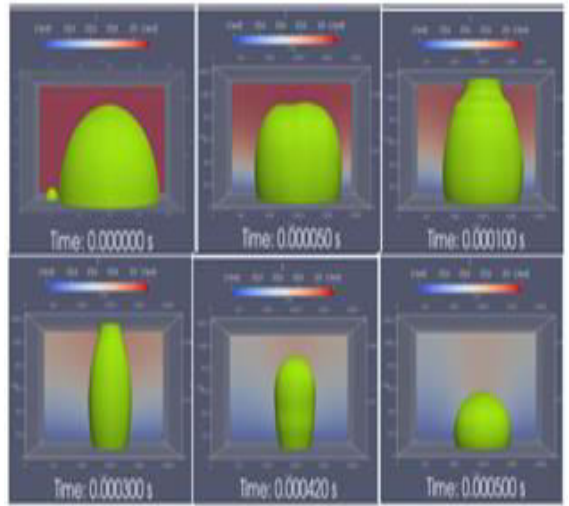

Fig 13: Effect of different contact angle and different radius of droplets for Case 9.

3.2 Effect of different surface wettability with dif- altered wettability's of the condense surface and ferent gravity vector orientation-Series $B$

In this section of the paper, the effect of gravity vector on the droplets coalescing on surfaces with different wettability characteristics is investigated numerically. For this, cases 1-9 from series (A) with various radii of droplets were utilised, but with an additional gravitational vector. Simulation series B, three sets of simulations with nine cases in total were carried out varying parameters explained in Table 2. The results of the simulations were as follows;
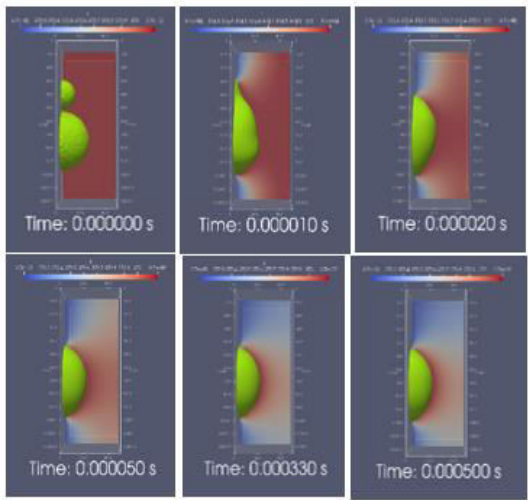

Fig 14: Effect of different contact angle and different radius of droplets for Case $1 b$.

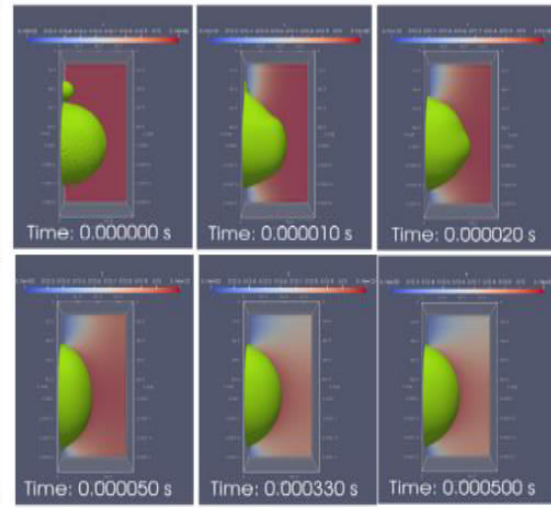

Fig 15: Effect of different contact angle and different radius of droplets for Case $2 b$.
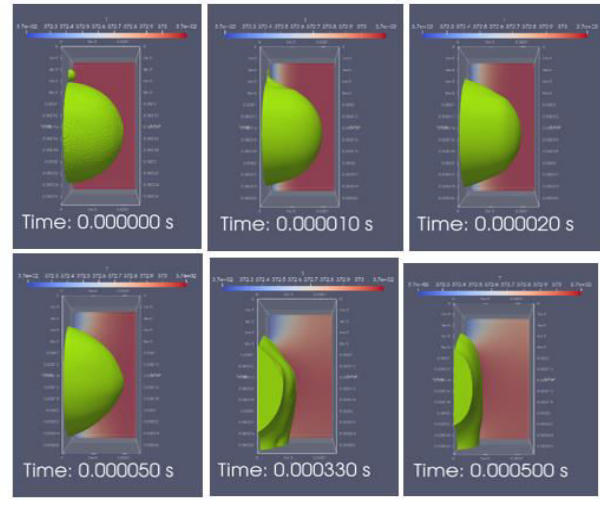

Fig 16: Effect of different contact angle and different radius of droplets for Case 3b.
In summary, Series B, set 1 Case $1 \mathrm{~b}-3 \mathrm{~b}$ carried out with same parameters as Set 1, Series A. wettability of the surface was set to hydrophilic conditions utilising the values found in Table 2. An additional gravity vector is applied symmetrically in $\mathrm{x}$-direction of the domain. The simulation results obtained are presented by rotating 90deg in order to show the relationships between gravity, surface tension and inertial forces. It can be observed, that case $1 b$ to $3 b$ undergoes a very similar process to case 1-3 in series A. droplets merges together and forms singular droplet with larger mass and condense gradually. However, small differences in values can be found when the values are observed carefully. Fig 17-21 illustrates that these cases $4 \mathrm{~b}$ to $9 \mathrm{~b}$ simulations undergoes very similar processes of coalescence as the series A. Case $4 b-6 b$ droplets coalescence and take off from the condensing surface by the momentum created. Similarly in cases $7 b-9 b$ droplets merge and condense gradually. In summary, as the results found in both series of simulations are similar, this shows that for the considered time scale gravity orientation does not play a major role, whereas the surface tension and inertial forces of the phenomenon are much more important for the considered spatial and temporal scale. 


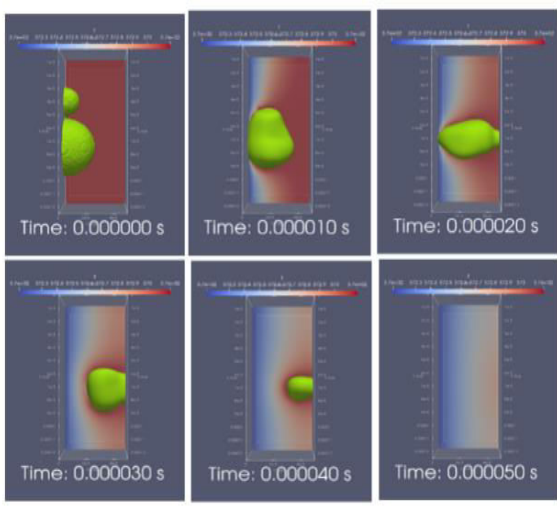

Fig 17: Effect of different contact angle and different radius of droplets for Case 4b.

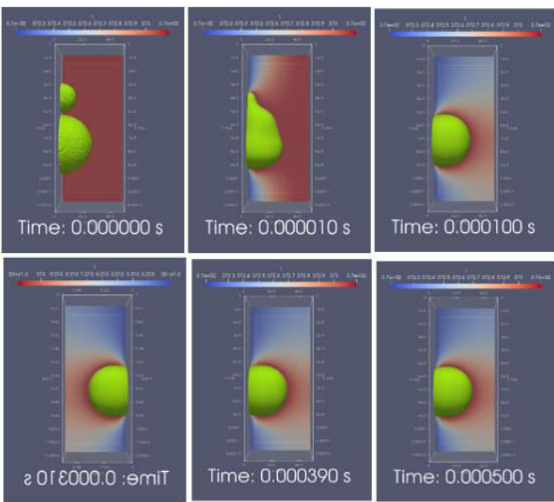

Fig 20: Effect of different contact angle and different radius of droplets for Case $7 b$.

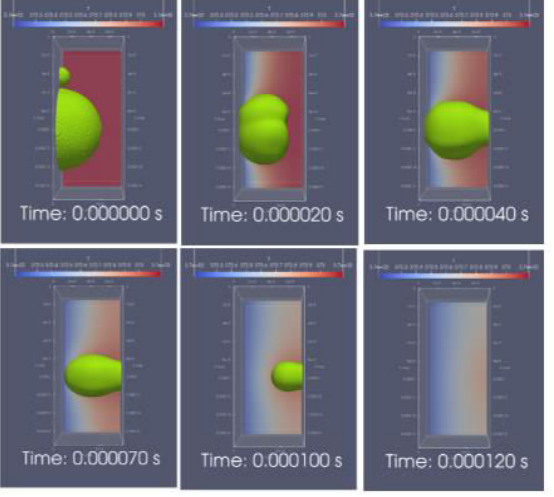

Fig 18: Effect of different contact angle and different radius of droplets for Case $5 \mathrm{~b}$.

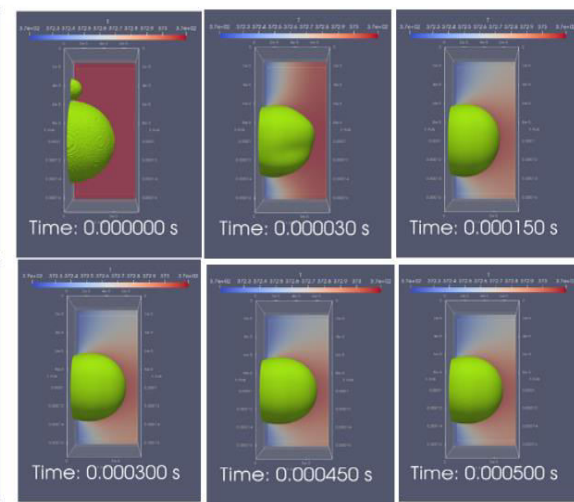

Fig 21: Effect of different contact angle and different radius of droplets for Case 8b.

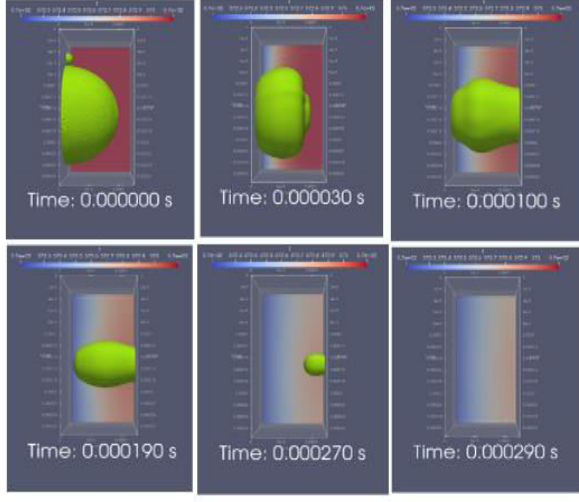

Fig 19: Effect of different contact angle and different radius of droplets for Case $6 b$.

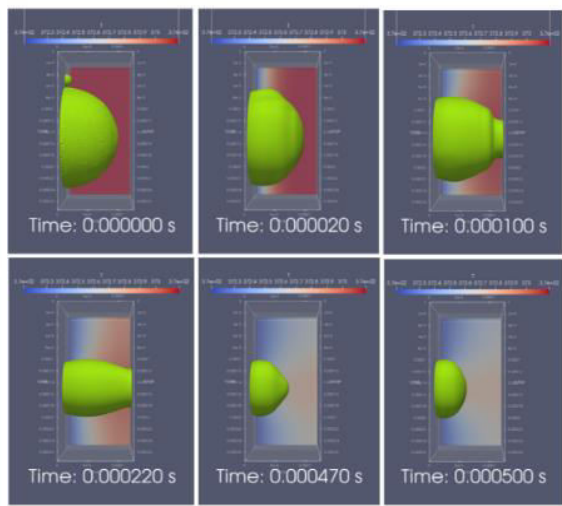

Fig 22: Effect of different contact angle and different radius of droplets for Case $9 b$.
3.3 Effect of different wettability characteristics on condensation rates of the phenomenon

In this section of the present paper, the results obtained in simulations series A, are examined in a more quantitative manner by comparing the volume integrals of transient condensation rates against time.

\subsubsection{Effect on the volume integral of condensation rate of droplets with different Radii}

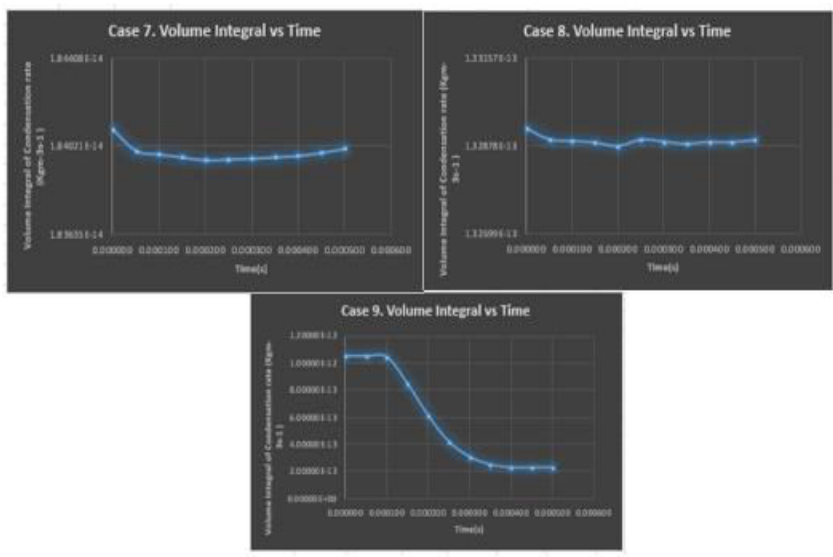

Fig 23: illustrates the effect on condensation rates of the phenomena with varied droplet radii on hydrophilic surface.
The ascending gradient shown in Case 1-2 shows that the volume of the droplet increases and stay steady as it undergoes coalesces process, meaning no condensation in the given time scale. Case 3 however shows slight increase volume, then rapid decrease in the gradient means decrease of volume integral; hence faster condensation rate.
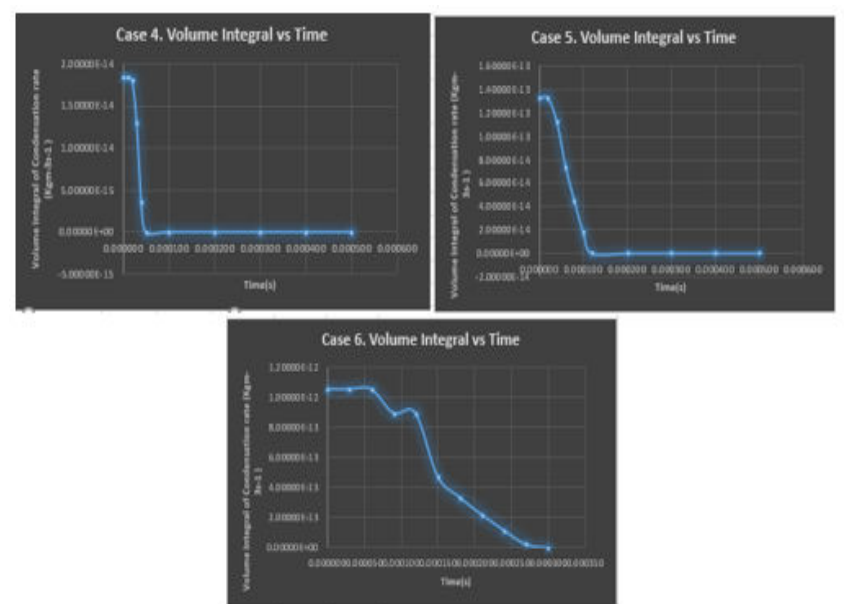

Fig 24: illustrates the effect on condensation rates of the phenomena with varied droplet radii on superhydrophobic surface. 


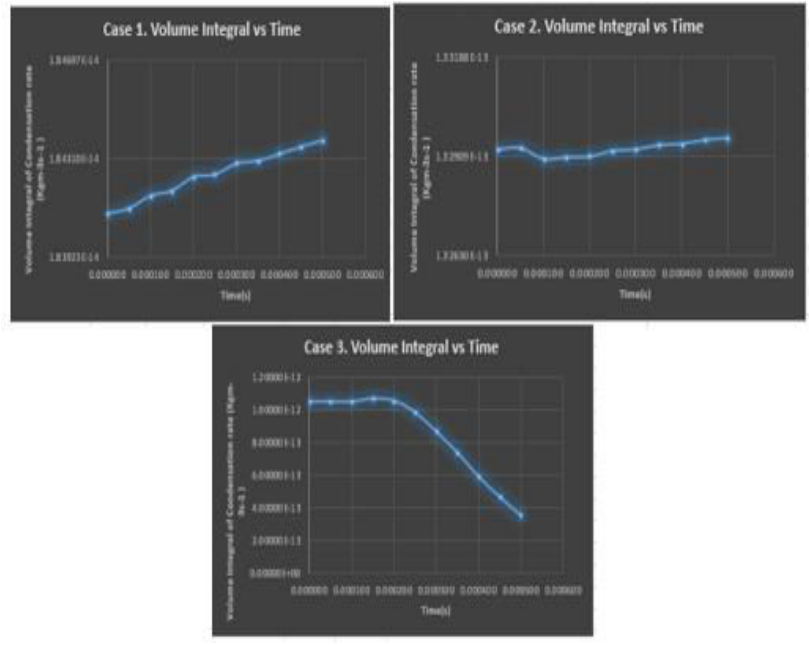

Fig 25: Illustrates the effect on condensation rates of the phenomena with varied droplet radii on hydrophobic surface.

Graphs in Fig 25 shows the two droplets with smaller radii lifts off $\mathrm{n}$ clear the surface with the rapidly decreasing gradient reaching $0 \mathrm{Kgm}-3 \mathrm{~s}-1$, meaning no or very little condensation. Graph for case 6 shows there is some unsteady change in the volume integral as the droplets merge and a change shape due to the momentum created, eventually lifts off and clears the surface.

From Fig 26, it can be obtained that once the droplets merge the volume integral does not change much in the cases $7 \& 8$. However, graph for case 9 shows how volume integral decreases over time; hence slowly condensing over time.

\subsubsection{Effect of the different wettability surfaces on volume integral of condensation rate}
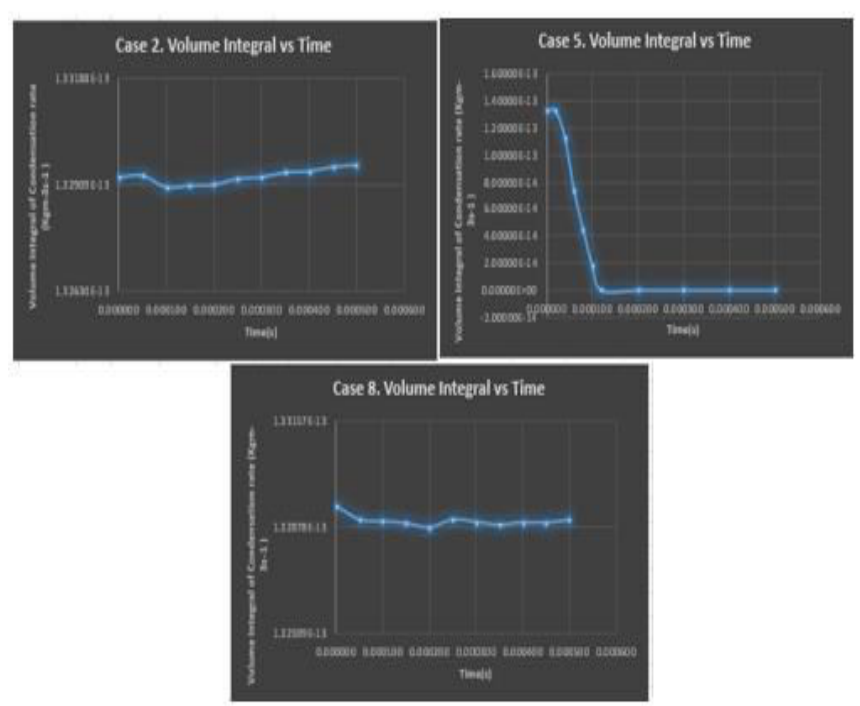

Fig 27: illustrates how different surface wettability effect the volume integral of condensation rate for droplets with radii of $12.5 \mathrm{um} \& 50 \mathrm{um}$.

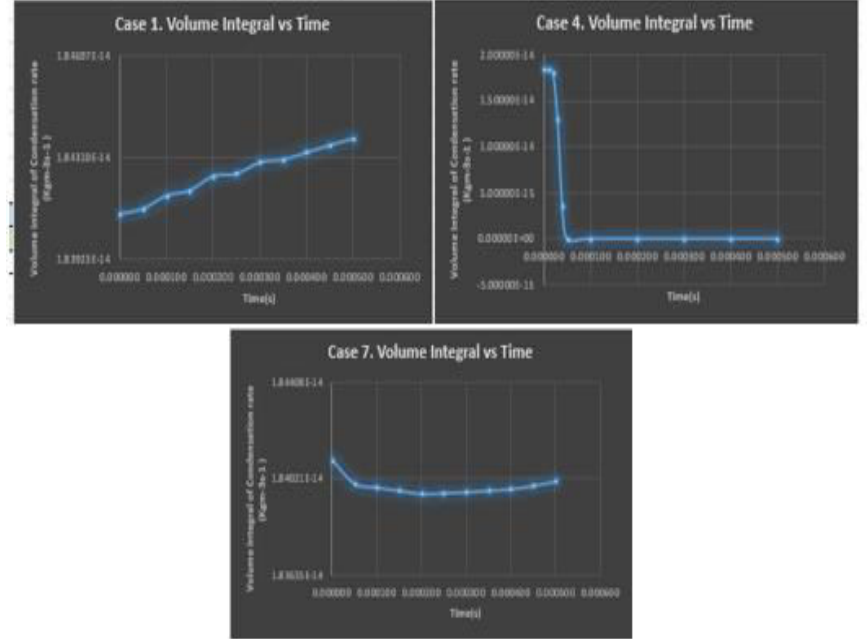

Fig 26: illustrates how different surface wettability affects the volume integral of condensation rate for droplets with radii of $12.5 \mathrm{um} \& 25 \mathrm{um}$.

In this section of the present paper, results obtained from simulations series (A) analysed in order to understand the effect of different wettability surfaces on the droplet condensation process. Simulations were performed in three sets. Radii of the droplets were kept constant and wettability of the surface is altered by varying the contact angle. Wettability of cases $1,4 \& 7$ changes from hydrophilic, superhydrophobic and hydrophobic respectively. Graph shows an increase in gradient for case 1, this means the two droplets merge and form a larger mass but does not go pass the primary mode of condensation. Case 4 shows rapid decrease in gradient and reaches 0 Kgm-3s-1 under 40us, hence the droplets has cleared off the domain.
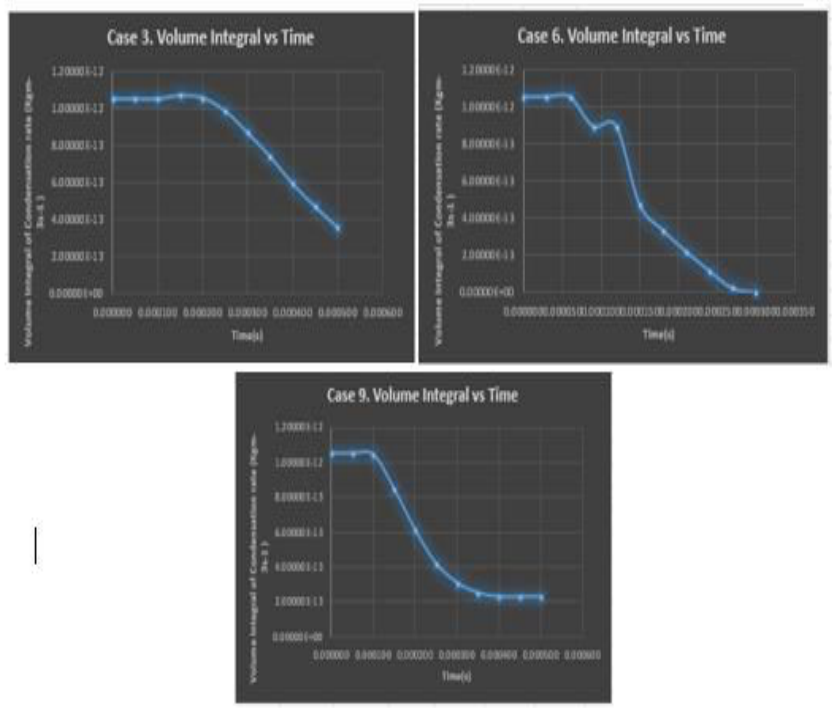

Fig 28: illustrates how different surface wettability effect the volume integral of condensation rate for droplets with radii of $12.5 \mathrm{um} \& 100 \mathrm{um}$. 
Fig 27 and 28 shows the results for cases with droplets radii of $12.5 \mathrm{um} \& 50 \mathrm{um}, 12.5 \mathrm{um}$ and $100 \mathrm{um}$ for the same wettability parameters discussed in Fig $\mathbf{2 6}$.

\subsection{Effect of different wettability characteristics on condensation rates of the phenomenon with a dif- ferent gravitational orientation.}

In this section of the present paper, the results obtained in simulations series B, are examined in a more quantitative manner by comparing the volume integrals of transient condensation rates against time.

\subsubsection{Effect on the volume integral of condensation rate of droplets with different Radii}

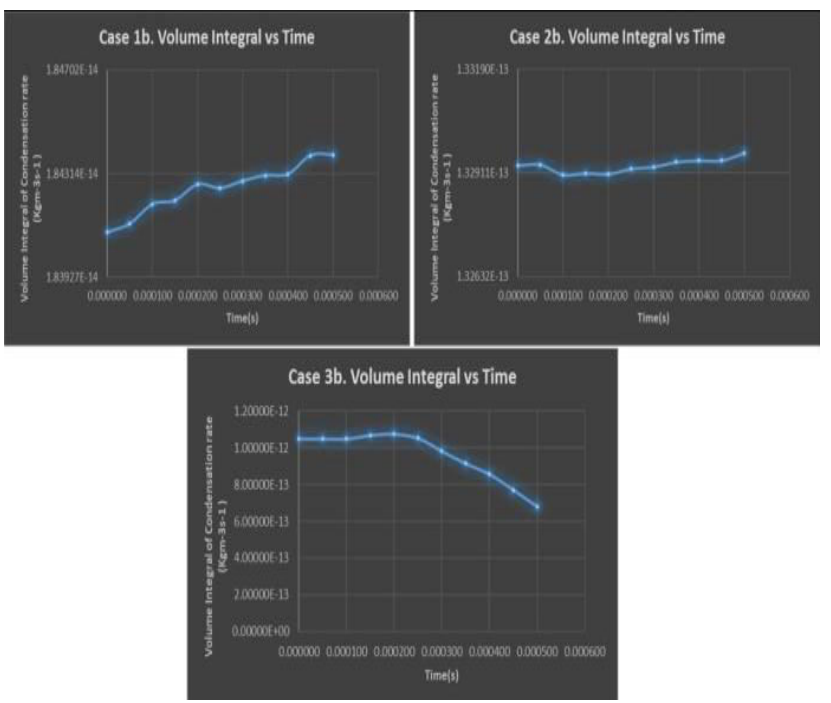

Fig 29: illustrates the effect on condensation rates of the phenomena with varied droplet radii on hydrophilic surface.
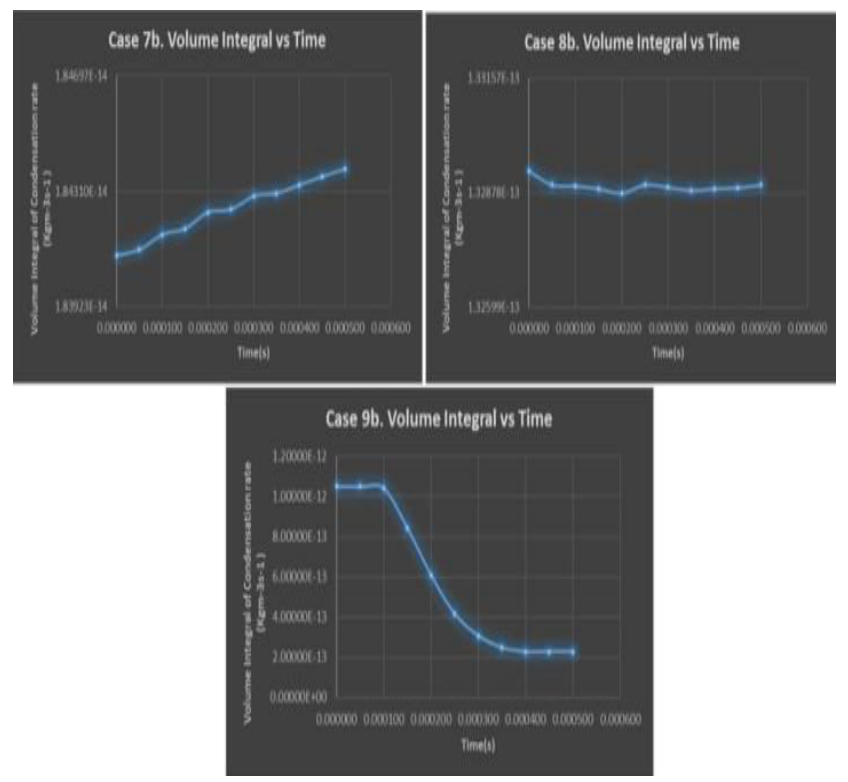

Fig 31: Illustrates the effect on condensation rates of the phenomena with varied droplet radii on hydrophobic surface.
Fig (29-34) shows the effect of gravity vector on the droplets coalescing on surfaces with different wettability characteristics. For this, cases 1-9 from series (A) with altered wettability's of the condense surface and various radii of droplets were utilised, but with an additional gravitational vector. As discussed earlier in results found in both series of simulations are similar, showing that for the considered time scale gravity orientation does not play a major role, where-as the surface tension and inertial forces of the phenomenon are much more important for the considered spatial and temporal scale (Uddin et al., 2021).

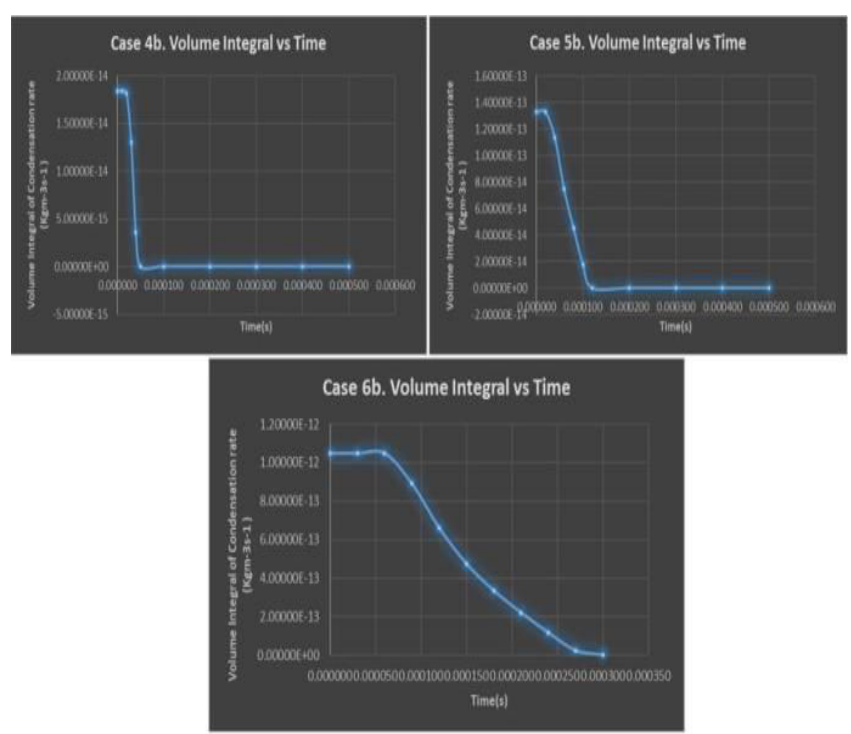

Fig 30: illustrates the effect on condensation rates of the phenomena with varied droplet radii on superhydrophobic surface
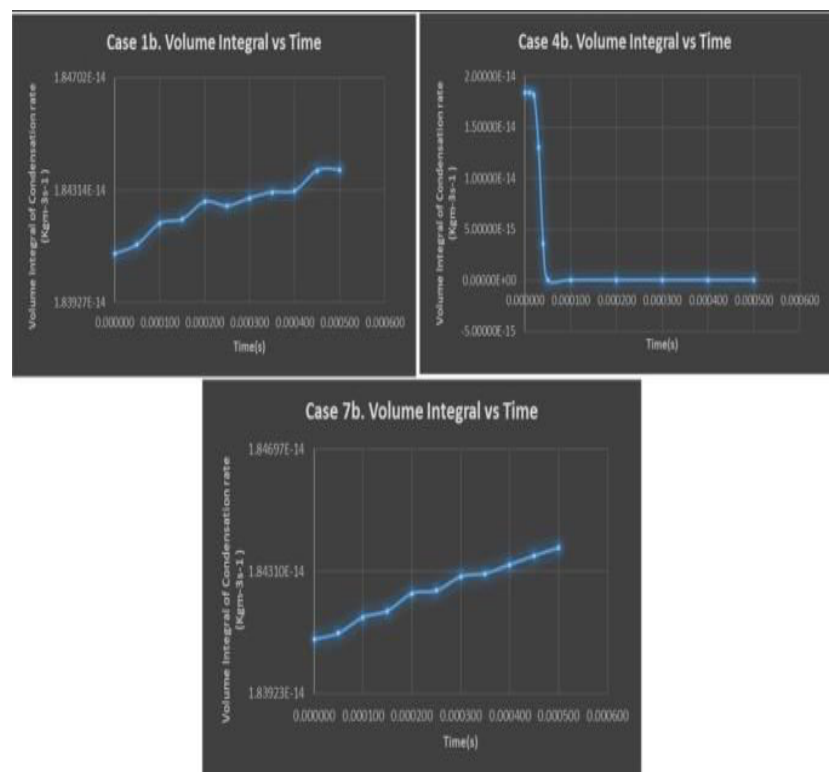

Fig 32: illustrates how different surface wettability effect the volume integral of condensation rate for droplets with radii of $12.5 \mathrm{um} \& 25 \mathrm{um}$. 


\subsubsection{Effect of the different wettability surfaces on volume integral of condensation rate.}
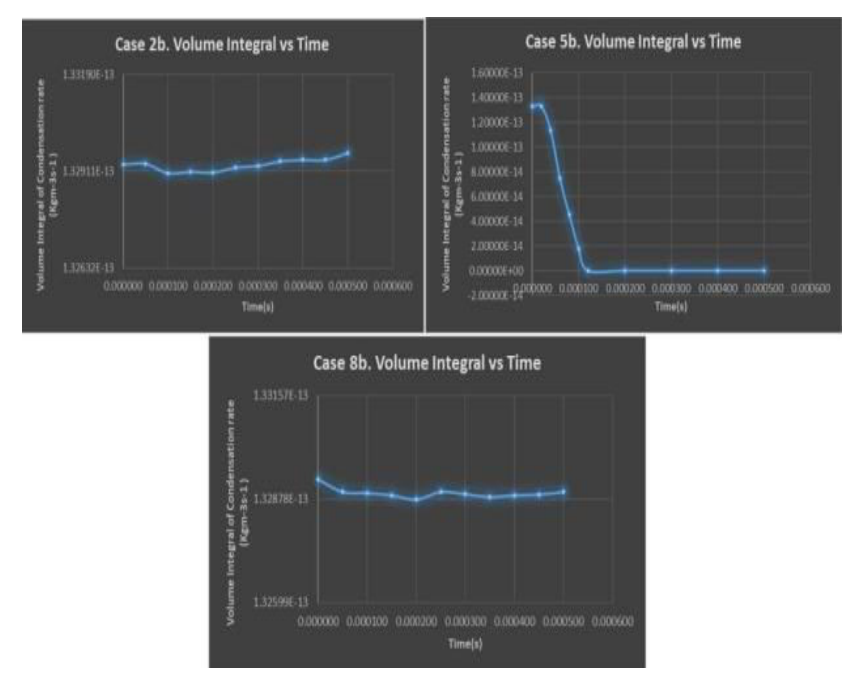

Fig 33: illustrates how different surface wettability effect the volume integral of condensation rate for droplets with radii of 12.5 um \& 50um.

\section{CONCLUSION:}

In this present work, an enhanced VOF based CDF model was used on a user modified solver of Open FOAM software, the solver was used and validated in the previous work done by Georgoulas et al. (Georgoulas et al., 2017). This modified solver was then applied to carry out series of simulations of the process of droplet coalesence in dropwise condensation. Simulations were carried out varying the wettability of the smooth plate to a given time scale. The effect of different parameters such as contact angle; advancing and receding angles, droplet radius and saturation temperature were investigated. Series $\mathrm{A}$ and $\mathrm{B}$ including a total of 18 cases over range of $(12.5 \mathrm{~mm} \leq \mathrm{r} \leq 100 \mathrm{~mm})$ and aspect ratio of $(2 \leq \mathrm{Rt} \leq 8)$. Simulation results then were used to understand the effect of different surface wettability in the initial stages (oms $\leq \mathrm{t} \leq 500 \mathrm{~ms}$ ) of dropwise condensation. The results were then further analysed in a quantitative manner by comparing the volume integral of condensation rate with time and the effect of droplet dynamics in the initial stages coalescence were investigated. Two main events occurred during the process. In some cases, the droplets merged and continued to condense. In other cases, due to momentum created by merging, droplets oscillated and lifted off from the condensing surface. These two events occurred depending on the characteristics of the surface. Simulations were successfully completed. Results from investigation gave a clear understating of the phenomenon during the initial stages of dropwise condensation for the given time scale as discussed in

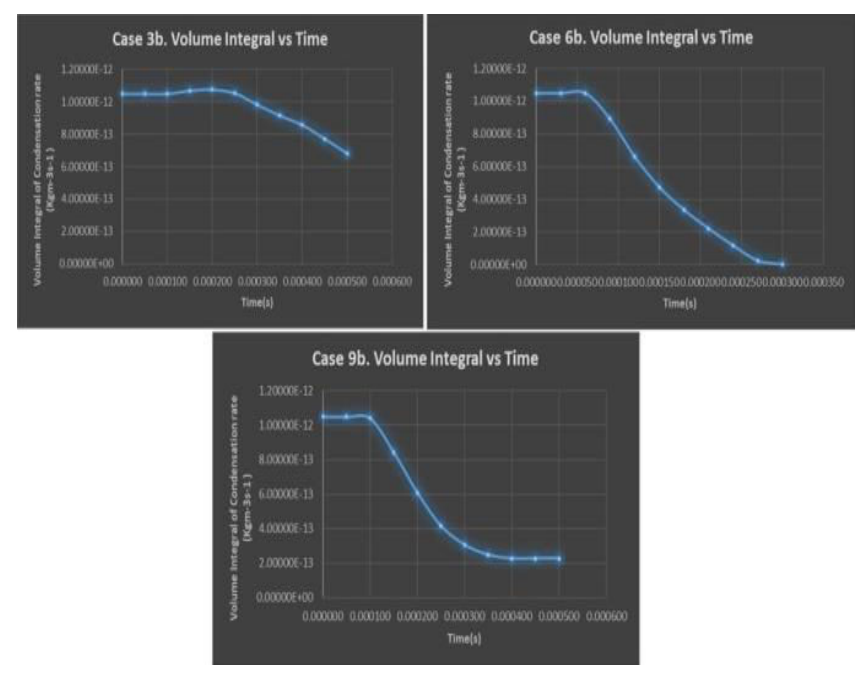

Fig 34: Illustrates how different surface wettability affects the volume integral of condensation rate for droplets with radii of $12.5 \mathrm{um} \& 100 \mathrm{um}$.

detail. Results from series A showed a significant effect of the droplets dynamics when surface wettability was varied. Results from simulation series B did now show major difference of the phenomenon when the gravity vector orientation is changed. To conclude, the results from present investigation help understand and adds to the existing knowledge of the fundamentals of coalescence of dropwise condensation.

\section{ACKNOWLEDGEMENT}

It gives me immense pleasure to have completed this dissertation and acknowledge the support and guidance that I have received throughout the process. I would like to thank my supervisor, Dr. Anastasios Georgoulas, who has not only guided me at every step of the project but has been extremely encouraging throughout. He has shown more confidence in me than I had myself. His eye for detail and constructive criticism has only pushed me to improve my work. I would also like to thank my fellow course mates, whose inputs have been insightful and whose support has been extremely comforting. In addition to this, I would like to thank my family for giving me the opportunity to pursue my passion and for patiently supporting me always. I hope I have managed to do justice to this project and am hopeful that this will be the beginning of many more projects.

\section{CONFLICTS OF INTEREST:}

The authors declared that there is no conflict of interest in this article. 


\section{REFERENCES:}

1) Antonini et al. (2013). "Drop Rebound after Impact: The Role of the Receding Contact Angle," Langmur, pp. 16045-16050.

2) Battoo et al. (2010). "Mathematical Modelling and Simulation of Dropwise Condensation and Inclined," Department of Mechanical Engineering Indian Institute of Technology Kanpur, Kanpur, India. https://doi.org/10.1007/s12046-013-0190-9

3) Burnside H. H. B.M., (1999). "Digital computer simulation of dropwise condensation from equilibrium droplet to detectable size," Int. J. Heat Mass transfer, p. 3137-3146. https://doi.org/10.1016/S0017-9310(98)00372$\underline{X}$

4) Chen R. P. J. W. S. G. X. (2016). "Coalescence-induced jumping of multiple condensate droplet on hierarchical super hydrophobic surfaces," Science Rep, 6: 18649. https://doi.org/10.1038/srep18649

5) Chu et al. (2012). "Relationship between condensed droplet coalescence and surface wettability," Heat Masss Transfer, pp. 836841.

https://www.scholarmate.com/F/cddf88303a51f a652c1e4c13955afd67

6) Deshpande et al. (2012). "Evaluating the performance of the two-phase flow solver inter Foam," Computational Science \& Discovery.

7) Enright N. M. J. S. K. N. R. M. R, (2014). "How coalescing droplets jump," ACS Nano, p. 10352-10362. https://doi.org/10.1021/nn503643m

8) Georgoulas et al. (2017). "An Enhanced VOF Method Coupled with Heat Transfer and Phase Change to Characterise Bubble," Energies, MDPI. 10(3), 272; https://doi.org/10.3390/en10030272

9) Hasan MR, Rahman KMR, Shohag MR, and Uddin MM. (2020). Efficient water management and selection of cooling system for future NPP in Bangladesh, Int. J. Mat. Math. Sci., 2(6), 93-98. https://doi.org/10.34104/ijmms.020.093098

10) Hoang et al. (2013). "Benchmark numerical simulations of segmented two-phase flows in micro channels using the Volume of Fluid method," Computers \& Fluids, 86(5), pp. 2836.

https://doi.org/10.1016/j.compfluid.2013.06.024

11) Incropera et al. (2002). In Fundamentals of Heat and Mass Transfer pp. 619-669.

12) Jalil MA., and Karami MJH. (2020). A critical exploration of some fundamental terms and terminologies in statistics, Int. J. Mat. Math. Sci., 2(3), 45-50.

https://doi.org/10.34104/ijmms.020.045050

13) Mei et al. (2009). "A fractal analysis of dropwise condensation heat transfer," International Journal of Heat and Mass Transfer. 52(21-22): 4823-4828.

https://doi.org/10.1016/j.ijheatmasstransfer.200 $\underline{9.06 .013}$

14) Mei B. Y. M. Z. L. L. M. (2013). "Numerical study on growth mechanism of dropwise condensation," Int. J. Heat Mass Transf., p. 54, 2004-2013.

15) OPENFOAM, (2019).

https://www.openfoam.com

16) Rattner A. S. and Adhikari S., (2018). "Heat transfer during condensing droplet coalescence," International Journal of Heat and Mass Transfer. 1, p. 3,

17) Rose J. (1981). "Dropwise condensation theory," International Journal of Heat and Mass Transfer, 19(12), pp. 191-194. https://doi.org/10.1016/0017-9310(81)90026-0

18) Sprittles J. and Shikhmurzaev Y., (2012). "Coalescence of liquid drops: different models," Physics of Fluids, 24, pp. 122105. https://doi.org/10.1063/1.4773067

19) Somwanshi et al. (2014). "Influence of Drop Shape and Coalescence on Dropwise," Proceedings of the $15^{\text {th }}$ International Heat Transfer Conference, pp. 1-20.

20) Uddin M, Uddin MM, and Khan MAH. (2021). Effect of shift parameters in rational Krylov subspace method for solving Riccati equations arise from power system models, Int. J. Mat. Math. Sci., 3(2), 43-49. https://doi.org/10.34104/ijmms.021.043049

21) Zemach et al. (1992). "A continuum method for modeling surface tension," journal of Computational Physics, 100(2), pp. 335-354. https://doi.org/10.1016/0021-9991(92)90240-Y 


\section{APPENDIX}

\subsection{Table of results from series A}
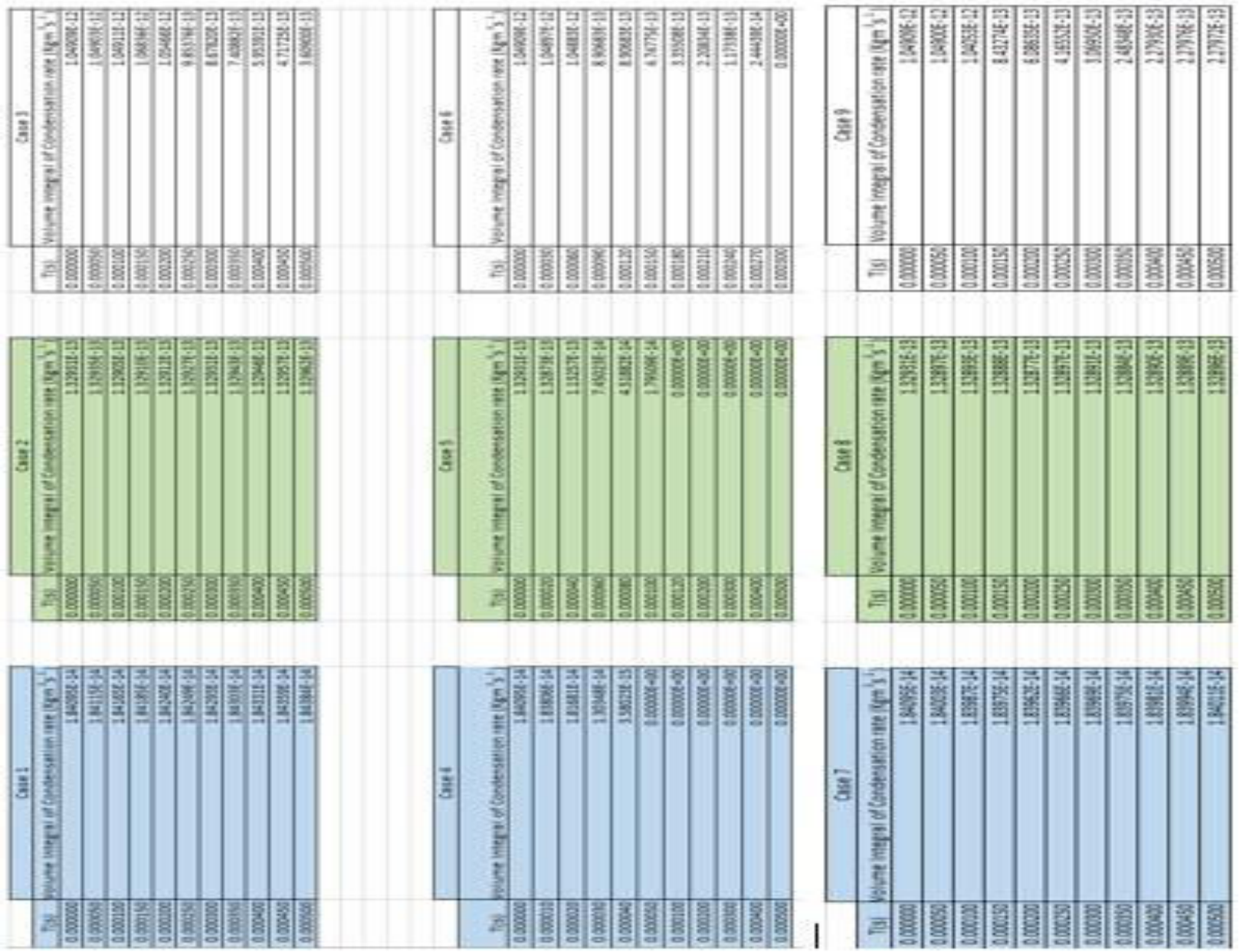

\subsection{Table of results from series $B$}
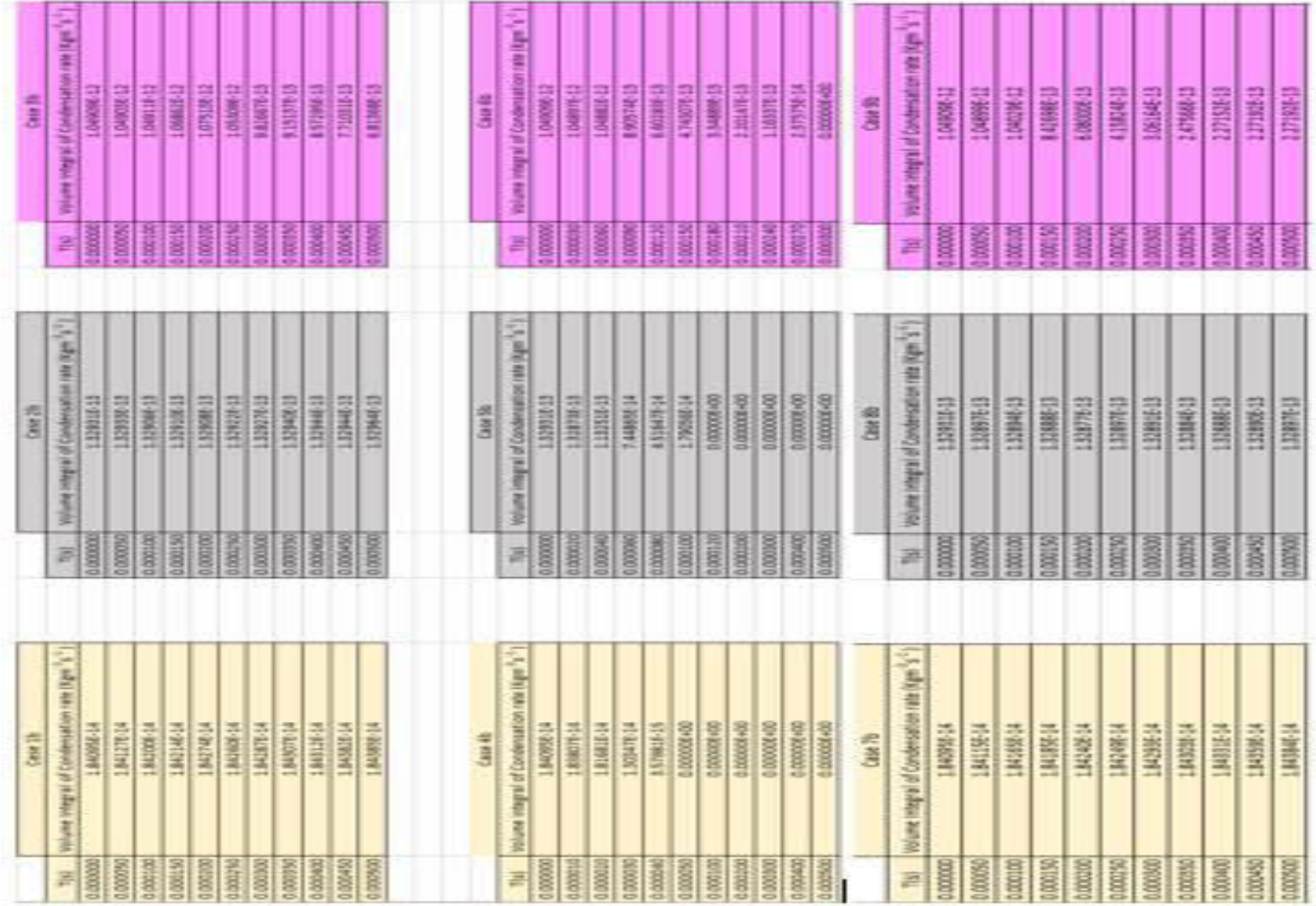


\subsection{RISK ASSESSMENT}

\begin{tabular}{|l|l|l|l|l|}
\hline School / Department: & Computing Engineering \& Mathematics & Date of assessment: & \multicolumn{2}{|c|}{ 21 $^{\text {st }}$ April 2017 } \\
\hline Activity / area: & Computer use in Computer Suites & Next review date: & $\begin{array}{c}20^{\text {th }} \text { April 2019 } \\
\text { or if procedure changes }\end{array}$ \\
\hline Assessed by: & Dave Stansbury & RA Ref No: & \\
\hline
\end{tabular}

\begin{tabular}{|c|c|c|c|c|c|c|c|c|}
\hline No. & $\begin{array}{l}\text { What are the } \\
\text { hazards? }\end{array}$ & $\begin{array}{l}\text { Who might be } \\
\text { harmed and } \\
\text { how? }\end{array}$ & $\begin{array}{l}\text { What controls do you } \\
\text { already have in place? }\end{array}$ & $\begin{array}{c}\text { Risk } \\
\text { (H/M/L) }\end{array}$ & $\begin{array}{l}\text { What further } \\
\text { action is } \\
\text { necessary to } \\
\text { reduce the risk to } \\
\text { Low? }\end{array}$ & $\begin{array}{l}\text { Action } \\
\text { by } \\
\text { whom? }\end{array}$ & $\begin{array}{l}\text { Action } \\
\text { by } \\
\text { when? }\end{array}$ & Done \\
\hline 1 & Trips and falls & Students and staff & $\begin{array}{l}\text { Training good housekeeping } \\
\text { and minimising the use of } \\
\text { trailing leads }\end{array}$ & L & None & & & \\
\hline 2 & Electrical shock & Students and staff & $\begin{array}{c}\text { Training and use of } \\
\text { standardised plug and socket } \\
\text { connections }\end{array}$ & L & None & & & \\
\hline 3 & Eye and muscle strain & Students and staff & $\begin{array}{l}\text { Take regular breaks - stand up } \\
\text { and move around / look at } \\
\text { something across the room } \\
\text { correct adjustment of seating }\end{array}$ & L & None & & & \\
\hline 4 & & & & & & & & \\
\hline 5 & & & & & & & & \\
\hline
\end{tabular}

\subsection{Gantt chart}

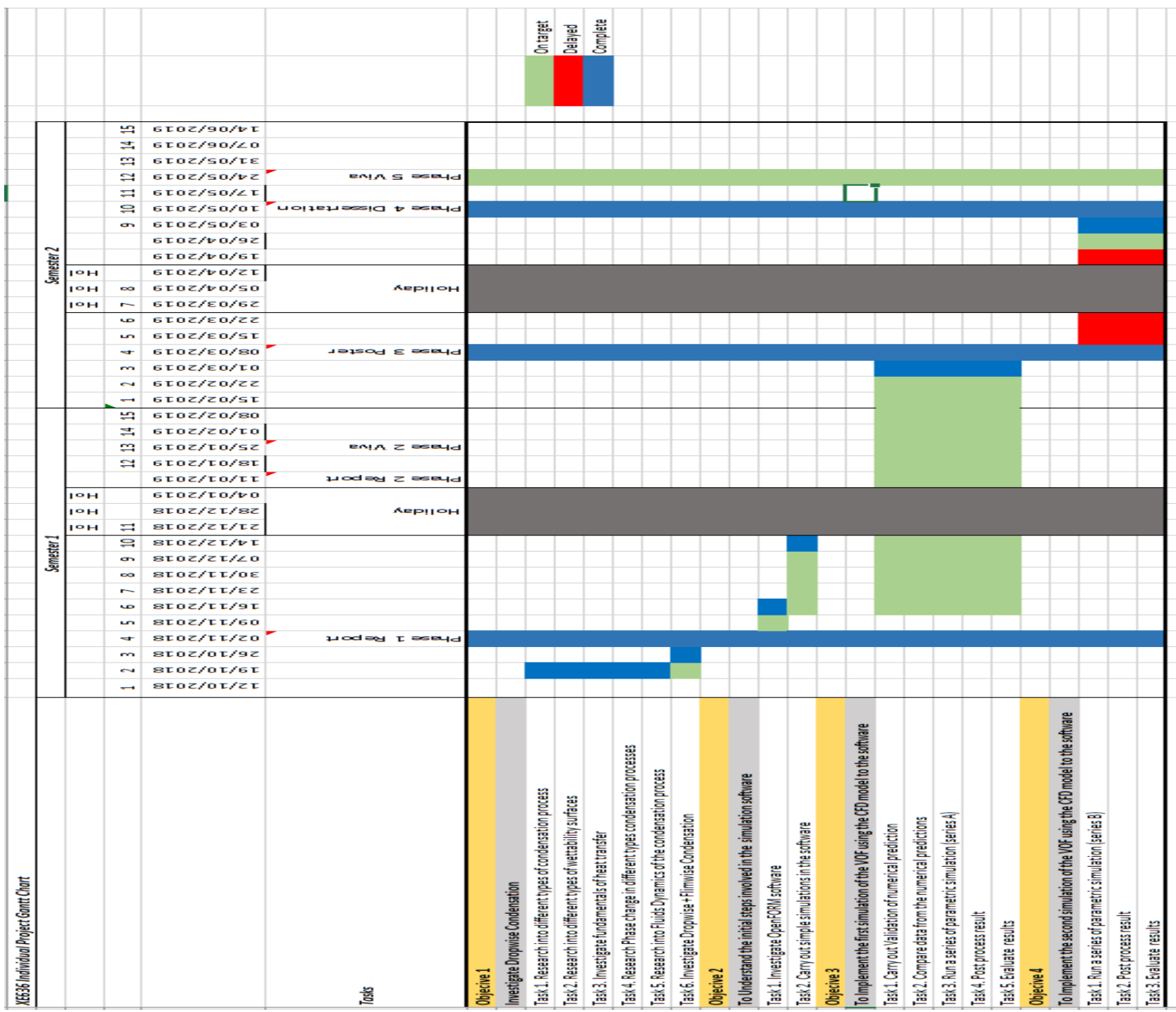

Citation: Pathiranage D. (2021). Numerical investigation of dropwise condensation on smooth plates with different wettability, Int. J. Mat. Math. Sci., 3(3), 60-73. https://doi.org/10.34104/ijmms.021.060073 @ @ 